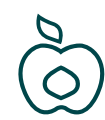

Nutrition and

Dietetics

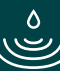

Impact Factor: $\mathbf{4 . 8 0 7}$

CiteScore: 7.3

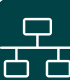

MEDLINE

\section{$?$}

DOAJ

\title{
Obesity Facts
}

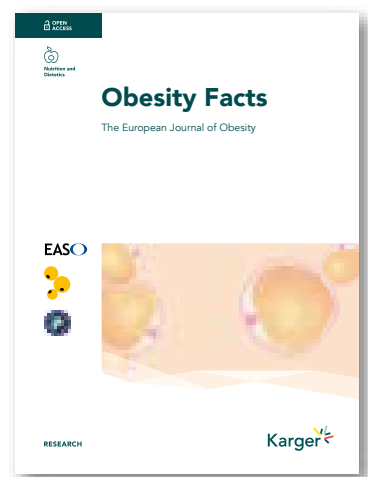

Editor-in-Chief

Hans Hauner (Munich)

The multidisciplinary online journal for obesity research and therapy

Obesity Facts publishes articles covering all aspects of obesity, in particular epidemiology, etiology and pathogenesis, treatment, and the prevention of adiposity. As obesity is related to many disease processes, the journal is also dedicated to all topics pertaining to comorbidity and covers psychological and sociocultural aspects as well as influences of nutrition and exercise on body weight.

The Official Journal of

karger.com

karger.com/ofa

Twitter
EASO

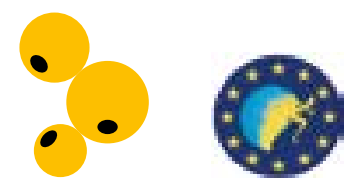

